\title{
Lentiviral-mediated siRNA targeted against osteopontin suppresses the growth and metastasis of gastric cancer cells
}

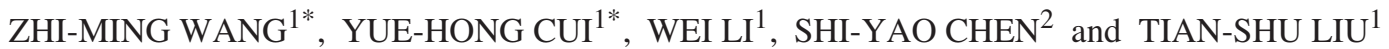 \\ Departments of ${ }^{1}$ Medical Oncology and ${ }^{2}$ Gastroenterology, Shanghai Zhongshan Hospital, \\ Fudan University, Shanghai 200032, P.R. China
}

Received October 13, 2010; Accepted December 16, 2010

DOI: 10.3892/or.2011.1168

\begin{abstract}
The expression of osteopontin (OPN) has been correlated with tumor growth and metastasis. However, the mechanisms by which OPN promotes tumor metastasis remain unclear. In this study, we aimed to investigate the anti-tumor effects of OPN by silencing OPN expression in the gastric cancer cell line SGC7901, using lentiviral-OPN small interfering RNA (siRNA) technology. Plasmid vectors containing OPN siRNAs were generated, encoded with lentiviral vector and transfected into SGC7901 cells (SGC$\mathrm{OPN}^{-}$cells). OPN mRNA and protein expression were examined using real-time reverse transcription polymerase chain reaction (RT-PCR) and Western blotting techniques. The tumorigenicity and metastatic potential of SGC7901 cells were studied in nude mice. Expression of OPN and vascular endothelial growth factor (VEGF) in lung metastatic tumor specimens were also examined using immunohistochemistry (IHC). Among the three siRNA sequences tested, siRNA2 most remarkably inhibited mRNA levels of OPN; lentiviral-siRNA2 was stably transfected into SGC7901 cells to generate SGC-OPN- cells. SGC-OPN- cells had significantly decreased OPN expression compared to control cells (relative intensities were $0.14 \pm 0.06$ vs. $0.95 \pm 0.16$ in controls, $\mathrm{P}<0.01)$. A substantial reduction in detectable tumors was found in mice implanted with $\mathrm{SGC}^{-\mathrm{OPN}^{-}}$cells compared to controls $\left(4.62 \pm 1.24\right.$ vs. $8.35 \pm 2.27 \mathrm{~cm}^{3}$ in controls, $\left.\mathrm{P}<0.01\right)$. In addition, mice implanted with $\mathrm{SGC}^{-} \mathrm{OPN}^{-}$cells survived longer $(101.2 \pm 22.5$ vs. $89.2 \pm 24.6 \mathrm{~d}, \mathrm{P}<0.01)$ and were demonstrated to have less metastases compared to mice implanted with SGC7901 control cells. Interestingly, lentiviral-siRNA2 also suppressed the expression of OPN and VEGF in metastatic lung specimens. Lentiviral-mediated OPN siRNA significantly reduced OPN gene expression,
\end{abstract}

Correspondence to: Dr Tian-Shu Liu, Department of Medical Oncology, Shanghai Zhongshan Hospital, Fudan University, Shanghai 200032, P.R. China

E-mail: tsliuceu@gmail.com

*Contributed equally

Key words: gastric cancer, osteopontin, small interfering RNA, vascular endothelial growth factor suppressing the growth and metastasis of gastric cancers, which might be related to reduced expression of VEGF. Therefore, OPN could serve as a promising therapeutic target for gastric cancer.

\section{Introduction}

Gastric cancer is the third most prevalent malignancy in the world and the second leading cause of cancer death, with nearly a million new cases diagnosed each year (1). Since the prognosis of gastric cancer depends largely on the stage at diagnosis, its high mortality is partially due to the fact that the disease has progressed to an unresectable stage at the time of diagnosis. The process of cell dissemination from primary tumors to distant sites is the most harmful event occurring during cancer progression, and the leading cause of death from cancer. Thus, understanding the precise mechanisms by which gastric cancer spreads to distant sites are of great importance.

OPN, an extracellular matrix protein, is a secreted phosphoglycoprotein that binds to the receptors for integrin $\alpha_{v} \beta$ and CD44 families. Elevated expression of OPN has been associated with invasion, progression or metastasis of tumors (2). Current studies have also revealed that OPN plays an important role in gastric cancer (3-5). Efforts have been made to inhibit tumor progression and metastasis by targeting OPN. Blockade of OPN expression using siRNA effectively suppressed the metastasis of CT26 murine colon adenocarcinoma (6).

siRNA is a post-transcriptional gene silencing strategy that is specifically targeted for evolutionarily conserved sequences. Lentiviral vectors offer unique versatility and robustness as vehicles for gene delivery. They can transduce a wide range of cell types and integrate into the host genome in both dividing and post-mitotic cells, resulting in long-term expression of transgenes both in vitro and in vivo. To explore the role of OPN in the biological behavior of gastric cancer cell lines, we previously investigated OPN protein expression and the biological changes before and after OPN siRNA transfer in gastric cancer cell lines (7), and found that OPN siRNA could down-regulate OPN and inhibit proliferation, migration and invasion of the gastric cancer cell lines MKN28 and SGC7901. This is consistent with a previous report (8) and provides further evidence that OPN plays an important role in the growth and metastases of gastric cancer. 


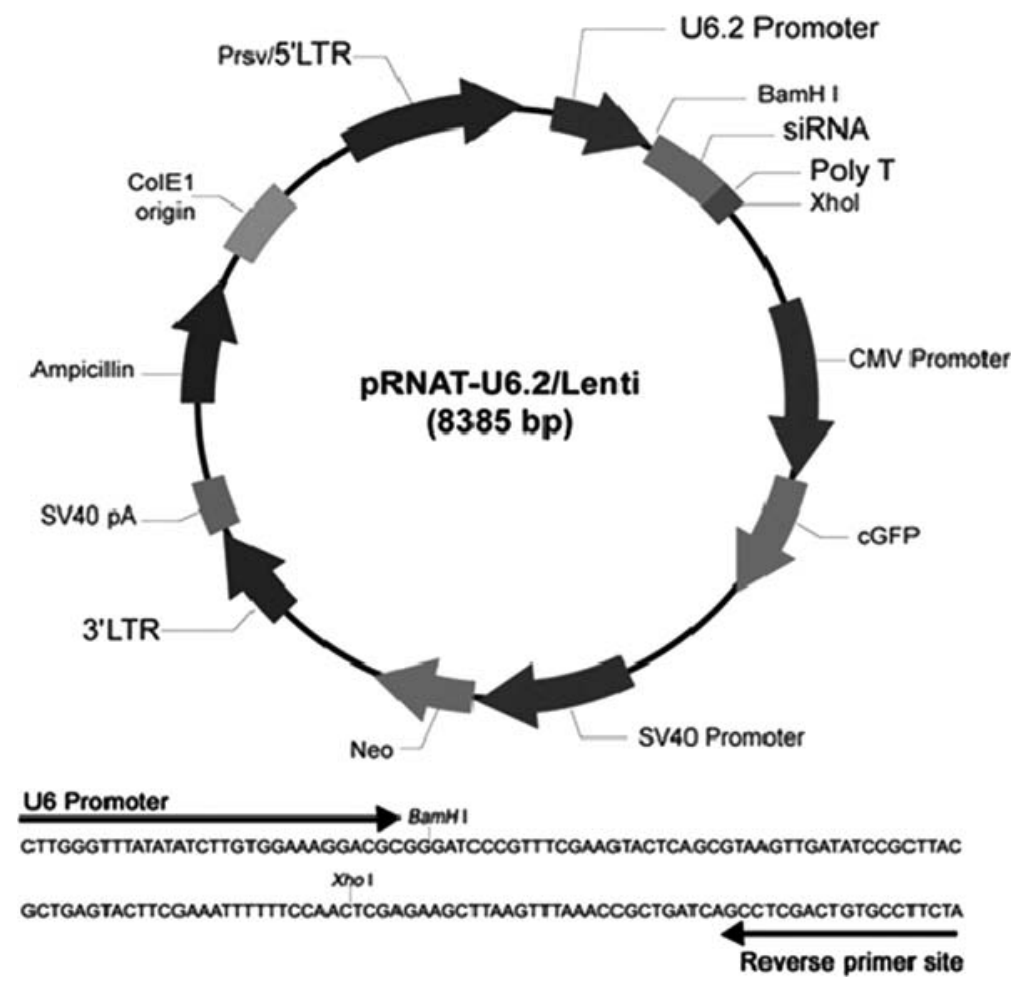

Figure 1. Diagram of pRNAT-U 6.2/Lenti vector used in the present study.

Recent studies have focused on the role of OPN in tumor angiogenesis (9-11). Both endogenous and exogenous OPN promote the expression of VEGF, a critical factor involved in tumor angiogenesis (12). In the present study, we hypothesize that overexpression of OPN in gastric cancer may be closely related to metastases and angiogenesis through regulation of VEGF expression. We used the powerful lentiviral-siRNA expression vectors with specific siRNA targeted against OPN to down-regulate OPN expression, and explored the preliminary correlation between OPN and VEGF in the metastases of gastric cancer.

\section{Material and methods}

Cell line. SGC7901 cells (obtained from Dr X. Zhang, the Department of Cancer Research, Chinese Medical University, Shenyang, China) were cultured in RPMI-1640 medium (contained $1.2 \mathrm{mg} / \mathrm{ml} \mathrm{NaHCO} 3,1 \times 10^{5} \mathrm{U} / 1$ penicillin and $1 \mathrm{mg} / 1$ streptomycin) supplemented with $10 \%$ fetal bovine serum (Gibco, Grand Island, NY, USA). Cells were cultured in an incubator at $37^{\circ} \mathrm{C}$ with $5 \% \mathrm{CO}_{2}$, and media were changed every 2-3 days. The basic growth patterns of the cells were monitored.

Construction of specific siRNAs against OPN. Three precursor siRNAs (pre-siRNA) sequences targeting OPN (Gen-Bank accession no. NM-000582) and a negative sequence for control were designed and approved by BLAST software. The four sequences were as followings: negative, 5'-CATGGAGT GGCACGTAGGT-3'; siRNA1, 5'-TAGACCCCATGGAGT GGCA-3; siRNA2, 5'-GGTGGCACAGCCACTGCAC-3'; siRNA3, 5'-ACCGTCTGGAGGCTGTTCA-3'. By utilizing these four DNA sequences, four plasmids were generated: pRNAT-negative, pRNAT-siRNA1, pRNAT-siRNA2 and pRNAT-siRNA3. Transfection of the plasmids was performed when the density of SGC7901 cells reached 80-90\% in 6well tissue culture plates. There were 5 groups: group A (control, Lipofectamine 2000 only), group B (pRNATnegative), group C (pRNAT-siRNA1), group D (pRNATsiRNA2) and group E (pRNAT-siRNA3). The expression levels of OPN mRNA were determined by RT-PCR.

Package of lentiviral vectors, measurement of lentiviral titer and identification of lentiviral by PCR. Double-stranded oligonucleotides encoding the siRNA2 sequence and its negative sequence were annealed and inserted into pRNAT-U 6.2/Lenti expression vector between BamHI (GGATCC) and XhoI (CTCGAG) (Fig. 1) using standard cloning procedures (GeneScript Corp., Piscataway, NJ, USA). The generated siRNA expression vectors were transfected into $293 \mathrm{~T}$ cells using lipofectamine 2000 (Fig. 2) for 8-10 days. The culture supernatant was collected when transfected 293T cells swelled and died and used as a virus stock. The viral titer was $5 \times 10^{10} \mathrm{pfu} / \mathrm{l}$ (determined by counting GFP-positive cells following transfection). RNA was extracted using the Rneasy mini kit (Qiagen, Hilden, Germany) from the purified virus stock and assessed using PCR in 1\% agrose electrophoresis (Fig. 3).

Generation and assessment of stable transfected cells. The lentiviral vectors were transfected into SGC7901 cells with the multiplicity of infection (MOI) of 50. Seventy-two hours following transfection, the cells were selected using $400 \mu \mathrm{g} /$ $\mathrm{ml} \mathrm{G} 418$. When the control cells were all dead and the 
Normal 293T cell Transfected 293T cells expressed GFP

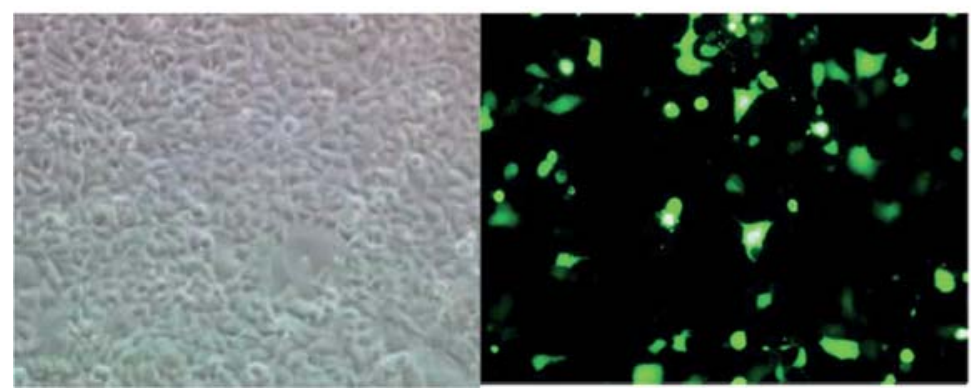

Figure 2. Transfection efficiency via lentiviral vectors. $293 \mathrm{~T}$ cells were transfected with lentiviral vectors expressing enhanced green fluorescent protein (GFP), which allowed for titering and measuring their infection efficiency in transfected cells.

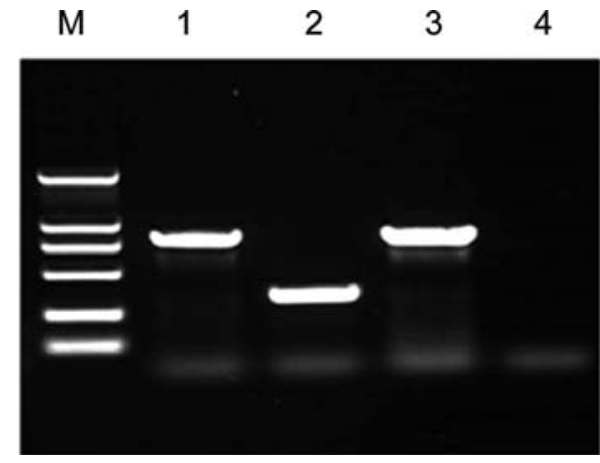

Figure 3. Identification of lentiviral expression by PCR. Lane M, marker; Lane 1, pRNAT-siRNA2 recombinant plasmid control (positive control); Lane 2, pRNAT plasmid control; Lane 3, pRNAT-siRNA2 recombinant lentiviral vectors; Lane 4, $293 \mathrm{~T}$ cells lysates (negative control).

experimental cells grew into distinctive clonies, the experimental cells were selected and transferred into culture flasks and were continuously selected with G418 for 5 weeks to generate stable recombinant cells, which were denoted SGC-OPN' and SGC-negative, respectively. The stable recombinant cells were cultured in RPMI-1640 medium containing $200 \mu \mathrm{g} / \mathrm{ml} \mathrm{G} 418$.

The expression levels of OPN-mRNA and glyceraldehyde3-phosphate dehydrogenase (GAPDH), an internal control, were evaluated by RT-PCR. The PCR primers were designed using Olig5 software and synthesized by the Shanghai Biosynthesis Co. The primer sequences of the OPN gene were designed with a forward primer of 5'-CCACATGGCTAAAC CCTGACC-3' and a reverse primer of 5'-CATGGCTTTCGT TGGACTTACTTG-3'. The product was $126 \mathrm{bp}$. The internal control GAPDH gene had a forward primer of 5'-ATGACCC CTTCATTGACC-3' and reverse primer of 5'-GAAGATGGT GATGGGATTTC-3'. Experiments were repeated independently for three times.

Western blotting was used to test the protein levels of OPN and B-actin, an internal control, using primary antibodies (OPN, R\&D Systems, Minneapolis, MN; ß-actin, Protein Tech, Chicago, USA). Total protein (5-50 $\mu \mathrm{g})$ extracted from the tested cells was separated by sodium dodecyl sulfatepolyacrylamide gel electrophoresis and transferred onto polyvinylidene fluoride membranes. Experiments were repeated independently three times.
In vivo assays of tumor growth and metastasis. Four- to sixweek-old female BALB/c nude mice were purchased from the Shanghai Experimental Animal Center of Chinese Academy of Sciences (Shanghai, China) and were fed in the Experimental Animal Center of Shanghai Traditional Chinese Medical University. All mice were maintained under pathogenfree conditions in laminar flow boxes in accordance with established institutional guidance and approved protocols. SGC-OPN- cells $\left(1 \times 10^{6}\right.$ at $\left.50 \mathrm{MOI}\right)$ were implanted subcutaneously into the flank of the nude mouse (6 mice in each group), and SGC7901 cells treated with phosphate-buffered saline were used as mock control. At day 28 following cell inoculation, the tumor volume was calculated according to the following formula: $\mathrm{V}\left(\mathrm{mm}^{3}\right)=$ length $(\mathrm{mm}) \mathrm{x}$ width ${ }^{2}\left(\mathrm{~mm}^{2}\right) / 2$. The rate of overall survival (OS) was calculated from the time of cancerous cell injection until the time of sacrifice when they were weak and dying, or until natural death. The mice were sacrificed for histopathological examinations, and the implanted tumors and lungs were stained with hematoxylin-eosin. The incidences of lung metastases was calculated and evaluated via microscopy. These procedures were approved by the Animal Care and Use Committee of Fudan University, China.

Histological and immunohistochemical examinations. For histological examinations, a portion of tissue was fixed with $10 \%$ neutrally buffered formaldehyde and was embedded in paraffin. The sections were deparaffinized and stained with hematoxylin and eosin. For immunohistochemical analyses, deparaffinized sections were immersed in $0.3 \% \mathrm{H}_{2} \mathrm{O}_{2}$ in PBS to eliminate endogenous peroxidase activities. The sections were further incubated with PBS containing normal serum derived from the same species, which were used to generate the secondary antibodies and $1 \%$ bovine serum albumin to reduce non-specific reactions. The sections were incubated with primary antibodies for anti-OPN (Santa Cruz Biotechnology, Santa Cruz, CA, USA) and anti-VEGF (Beyotime, Shanghai, China) at optimal concentrations at $4^{\circ} \mathrm{C}$ overnight. After incubation with biotinylated secondary antibodies at room temperature for $30 \mathrm{~min}$, the nuclei were stained with Hoechst (Sigma, St. Louis, MO, USA), immunoreactive cells were visualized using Catalyzed Signal Amplification System (Dako, Denmark) according to the manufacturer's instructions, and images were then captured with a microscope (Olympus BX51) equipped with a SPOT digital camera. 


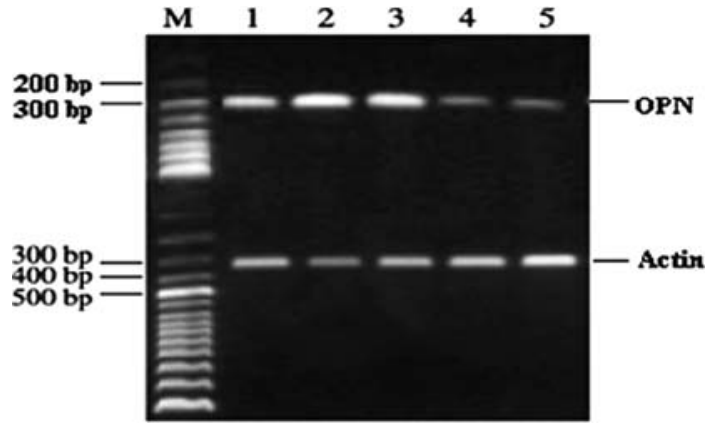

Figure 4. Inhibition of OPN mRNA expression. Lane M, marker; Lane 1, mock control (Lipofectamine 2000 only); Lane 2, transfection with pRNATnegative; Lanes 3-5, transfection with pRNAT-siRNA1, pRNAT-siRNA2 and pRNAT-siRNA3. Their relative intensities (OPN/ß-actin) were 2.14 , $1.82,1.93,0.36$ and 0.47 , respectively.

Statistic analysis. Statistical analyses were performed using Software SPSS 11.0. All data are expressed as the mean \pm standard deviation (SD), and analyzed using a Student's t-test. The level of significance was set at $\mathrm{P}<0.05$.

\section{Results}

Identification of specific and efficient siRNA sequence against OPN. Lipofectamine 2000 mediated siRNA delivery substantially inhibited mRNA expression of OPN in gastric cancer cells. As shown in Fig. 4, pRNAT-siRNA2 and pRNAT-siRNA3 demonstrated significant reductions in the mRNA level of OPN. As pRNAT-siRNA2 exhibited the most effective inhibition, it was then selected to be packaged into lentiviral vectors in the following experiments.

Identification of stable transfected cells and morphologic observation. Two stable recombinant cell lines were generated using pRNAT-siRNA2 and pRNAT-negative, which were denoted as SGC-OPN- ${ }^{-}$and SGC-negative, respectively. As shown in Fig. 5, the stable recombinant cells expressed enhanced GFP.

RT-PCR and Western blot analysis. To verify the expression of mRNA and protein levels of OPN in transfectants, we performed RT-PCR and Western blot analyses. As shown in Table I, the lowest level of OPN mRNA was found in SGC-OPN- cells, while no difference was found between SGC7901 and SGC-negative cells. The protein levels of OPN were further evaluated using Western blotting. As shown in Fig. 6, the OPN protein level was also significantly inhibited in SGC-OPN' cells, while no difference was found between SGC7901 and SGC-negative cells. We therefore utilized SGC-OPN- cells in subsequent experiments.

Effect of down-regulation of OPN on tumor growth and metastases of gastric cancer. To further investigate the effect of OPN down-regulation on tumor growth and metastases of gastric cancer, SGC-OPN- or SGC7901 cells were subcutaneously implanted into nude mice. Xenograft tumor masses were found in all mice, and the tumor length of the SGC7901 group was 2-3 $\mathrm{mm}$ at day 10 following cell innoculation. Smaller detectable tumor masses $\left(4.62 \pm 1.24 \mathrm{~cm}^{3}\right)$ were found in mice of the SGC-OPN ${ }^{-}$group, while much larger tumors were detected in mice in the SGC7901 group $\left(8.35 \pm 2.27 \mathrm{~cm}^{3}\right)$ $(\mathrm{P}<0.01)$ (Fig. 7).

To investigate lung metastases, consecutive sections were stained with hematoxylin and eosin for each lung tissue block. The incidences of lung metastases in the SGC7901 and SGCOPN' groups were $83.3 \%(5 / 6)$ and $0 \%(0 / 6)$, respectively, and the lung metastatic lesions in the SGC7901 group were multiple (Fig. 8).

Effect of OPN down-regulation on the survival of gastric cancer cells. Based on the effects of OPN down-regulation on the in vivo tumor growth and metastases of gastric cancer, the overall survival rate was assessed and compared in the

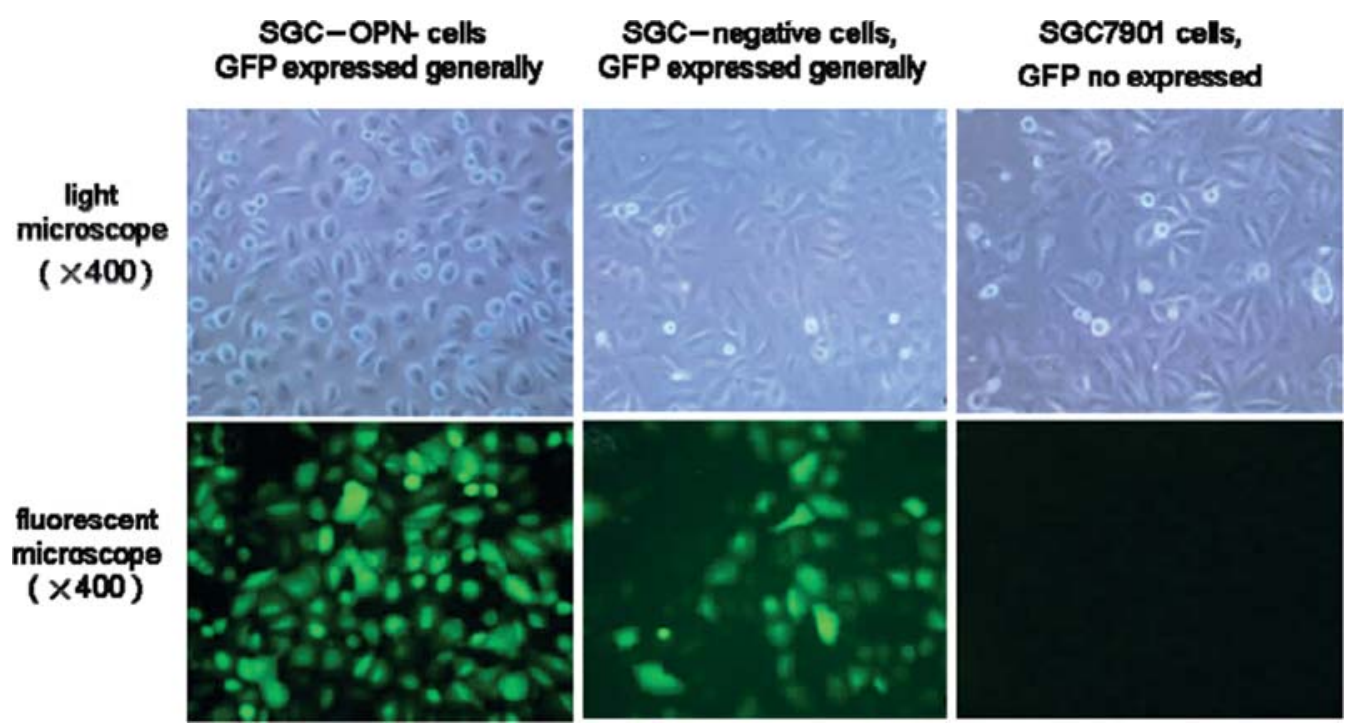

Figure 5. Morphological changes. The transfected cells were observed as a green color excited under flourescence microscopy in SGC-OPN- cells and SGCnegative cells, while GFP was not expressed in SGC7901 cells (all panels, x400). 
Table I. OPN mRNA expression evaluated via RT-PCR.

\begin{tabular}{|c|c|c|c|c|c|}
\hline Group & $\mathrm{Ct}$ & Copies/ $\mu 1$ (OPN) & Copies/ $\mu 1$ (GADPH) & Copies/10 6 GAPDH & Mean copies $/ \mu 1$ \\
\hline \multirow[t]{3}{*}{ SGC7901 } & 11.24 & 78.297 & $9.24 \mathrm{E}+04$ & $8.47 \mathrm{E}+05$ & $7.70 \mathrm{E}+05$ \\
\hline & 11.54 & 69.178 & $9.26 \mathrm{E}+04$ & $7.47 \mathrm{E}+05$ & \\
\hline & 11.81 & 67.069 & $9.38 \mathrm{E}+04$ & $7.15 \mathrm{E}+05$ & \\
\hline \multirow[t]{3}{*}{ SGC-negative } & 11.15 & 63.592 & $9.30 \mathrm{E}+04$ & $6.84 \mathrm{E}+05$ & $6.97 \mathrm{E}+05$ \\
\hline & 11.77 & 68.247 & $9.57 \mathrm{E}+04$ & $7.13 \mathrm{E}+05$ & \\
\hline & 11.67 & 66.514 & $9.56 \mathrm{E}+04$ & $6.96 \mathrm{E}+05$ & \\
\hline \multirow[t]{3}{*}{ SGC-OPN- $^{-}$} & 20.2 & 1.937 & $9.38 \mathrm{E}+04$ & $2.07 \mathrm{E}+04$ & $1.49 \mathrm{E}+04$ \\
\hline & 21.11 & 1.327 & $9.78 \mathrm{E}+04$ & $1.36 \mathrm{E}+04$ & \\
\hline & 21.81 & 996 & $9.37 \mathrm{E}+04$ & $1.06 \mathrm{E}+04$ & \\
\hline
\end{tabular}

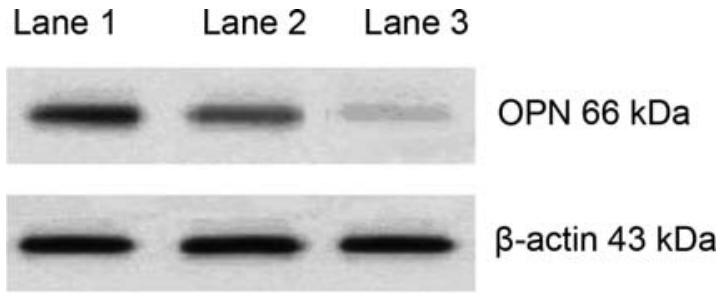

Figure 6. Western blot analyses. Lane 1, SGC7901 cells; Lane 2, SGCnegative cells; Lane 3, SGC-OPN ${ }^{-}$cells, of which relative gray scale was $0.95 \pm 0.16,0.84 \pm 0.20,0.14 \pm 0.06$, respectively. Data are presented as the mean \pm SD from three independent experiments $(\mathrm{P}<0.05)$.

SGC7901 and SGC-OPN' groups. The mice in the SGC-OPN group survived longer compared with mice in the SGC7901 group $(101.2 \pm 22.5$ vs. 89.2 $\pm 24.6 \mathrm{~d}, \mathrm{P}<0.01)$. Moreover, the mice in the SGC7901 group appeared sick. Therefore, we assumed that the mice in the SGC7901 group had reduced life time compared with the SGC-OPN- group.

Down-regulation of OPN suppressed the metastases of gastric cancer cells by inhibiting VEGF expression. To explore the possible mechanisms of OPN in regulating tumor growth and metastases, the expression levels of OPN and VEGF were investigated in lung metastases by IHC. Compared with controls, the expression levels of OPN and VEGF in lung tissue were both decreased significantly in mice of the SGC-OPNgroup (Fig. 9). Lentiviral-OPN siRNA2 significantly suppressed the expression of OPN and VEGF in lung specimens.

\section{Discussion}

In the present study, we designed three plasmid vectors expressing the specific sequences of siRNA against OPN (pRNAT-siRNA1, pRNAT-siRNA2 and pRNAT-siRNA3) and our data showed that pRNAT-siRNA2 most efficiently down-regulated OPN expression. Compared to control cells, OPN gene silencing of SGC-OPN ${ }^{-}$cells not only significantly inhibited in vivo tumor growth $\left(4.62 \pm 1.24\right.$ vs $8.35 \pm 2.27 \mathrm{~cm}^{3}$, $\mathrm{P}<0.01$ ), but also reduced lung metastases accompanied by decreased expression of VEGF, suggesting a close relationship between OPN and VEGF expression in SGC7901 cells.
It is well known that tumor angiogenesis is essential for the growth and metastases of solid tumors. Increased evidence has suggested that VEGF plays an important role in tumor angiogenesis and progression (13). Our present data therefore revealed that the OPN gene may promote tumor development by altering VEGF levels. OPN could be a promising target for the control of metastasis as well as gastric tumor growth or a hopeful target for anti-angiogenesis in gastric tumors. OPN protein or mRNA levels increased in many different types of tumors, including breast, liver and prostate cancers, and were also related to tumor progression, and metastases (14-16). Several studies have demonstrated that OPN plays an important role in the metastases and tumor growth of hepatocellular carcinoma (HCC) through activation of mitogen-activated protein kinase (MAPK), NF- $\mathrm{KB}$ and MMP-2 pathways (17). In gastric cancer BGC-823 cells, OPN targeted siRNA suppressed growth, migration and invasion through reduction in MMP-2 and UPA expression, inhibition of NF- $\mathrm{BB}$ DNA binding activity and down-regulation of Akt phosphorylation (8). Our present results provide further evidence that OPN might regulate tumor growth and metastases through alterations in VEGF levels. This is similar to a previous report (18) that reduced OPN production by gastric cancer cells could reduce the proliferation, migration and tube formation of human umbilical vein endothelial cells, and led to decreased angiogenesis in transplanted tumors of mice. These data confirmed the positive roles for OPN in the angiogenesis associated with gastric cancer.

No apoptosis was induced following transfection of Lipofectin-OPN siRNA in two gastric cancer cell lines in our previous study (7), implying that the necessary level of OPN for cell survival is much lower than that for the invasion and metastasis of SGC7901 cells, and a very low level of OPN is effective for cell survival. Therefore, in our in vivo experiment, apoptosis of cancer cells in xenograft tumors and lung metastases were not detected. A possible limitation of our experiment is that we only explored the preliminary relationship between OPN and VEGF expression in tumor tissues, and the possible mechanisms by which OPN was involved in down-regulation of VEGF requires further studies. Several OPN-downstream targets and OPN-dependent signaling pathways, such as uPA, MMP-2, MMP-9, MEK/ERK/1/2 


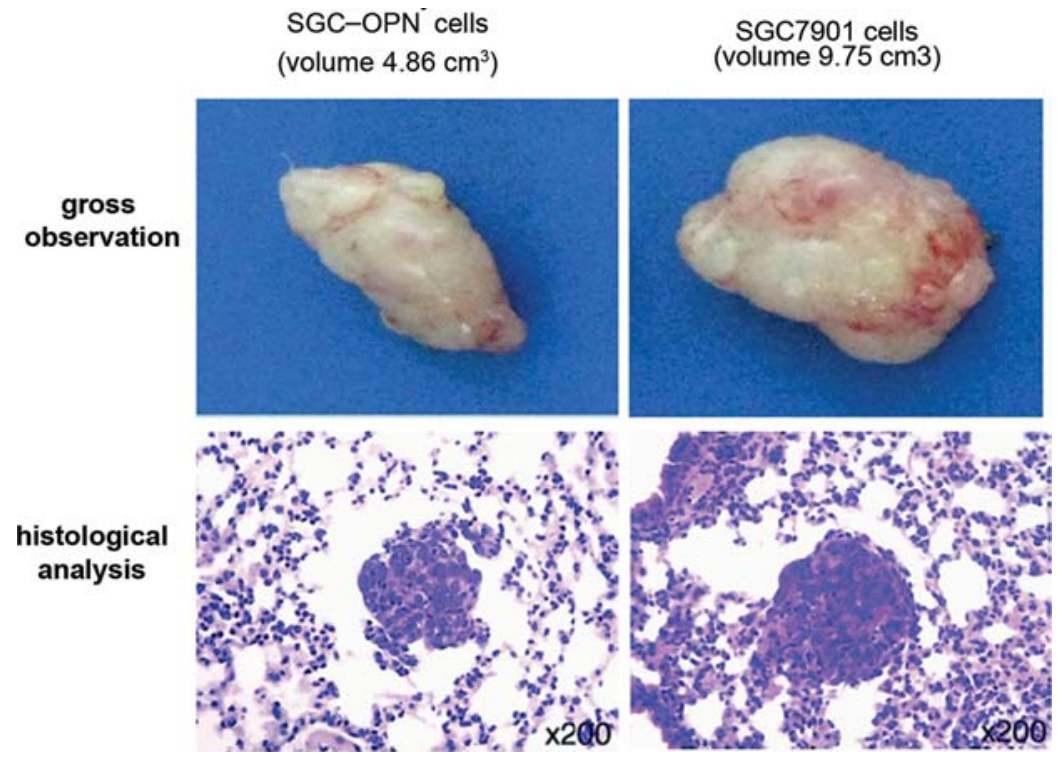

Figure 7. Effects of lentiviral-mediated siRNA against OPN on tumor growth in vivo. Representative data from histological analyses of tumor (stained with hematoxylin and eosin, magnification x200).

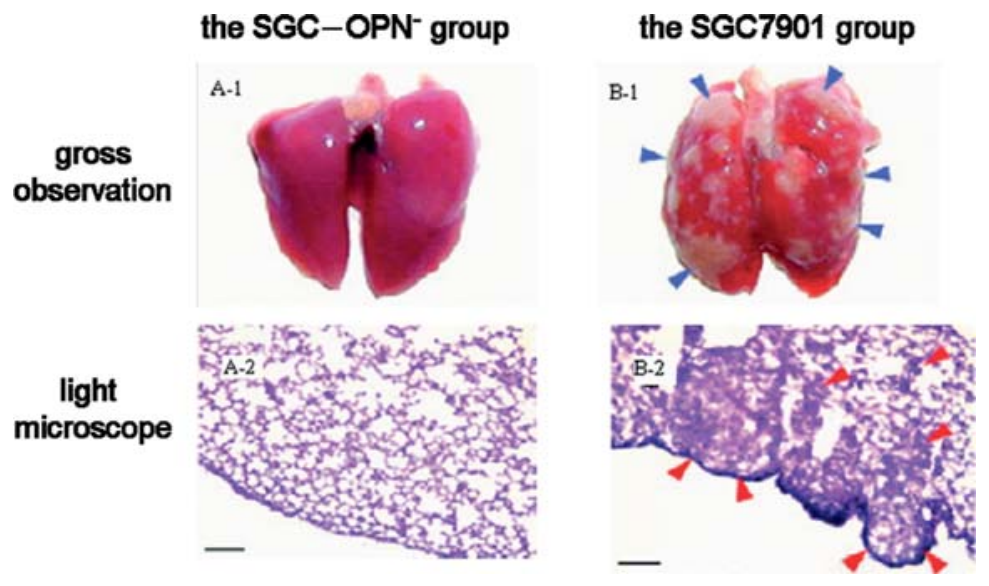

Figure 8. Lung metastases in mice. (A-1) and (A-2), the SGC-OPN- group; (B-1) and (B-2), the SGC7901 group. Tumor metastases were examined by gross observation (A-1, B-1) and H\&E staining (A-2, B-2). Bar, $120 \mu \mathrm{m}$. Grossly visible metastases (blue arrowheads) in the lung were obvious, and micrometastatic tumors were revealed by $\mathrm{H} \& \mathrm{E}$ staining (red arrowheads).

the SGC-OPN group

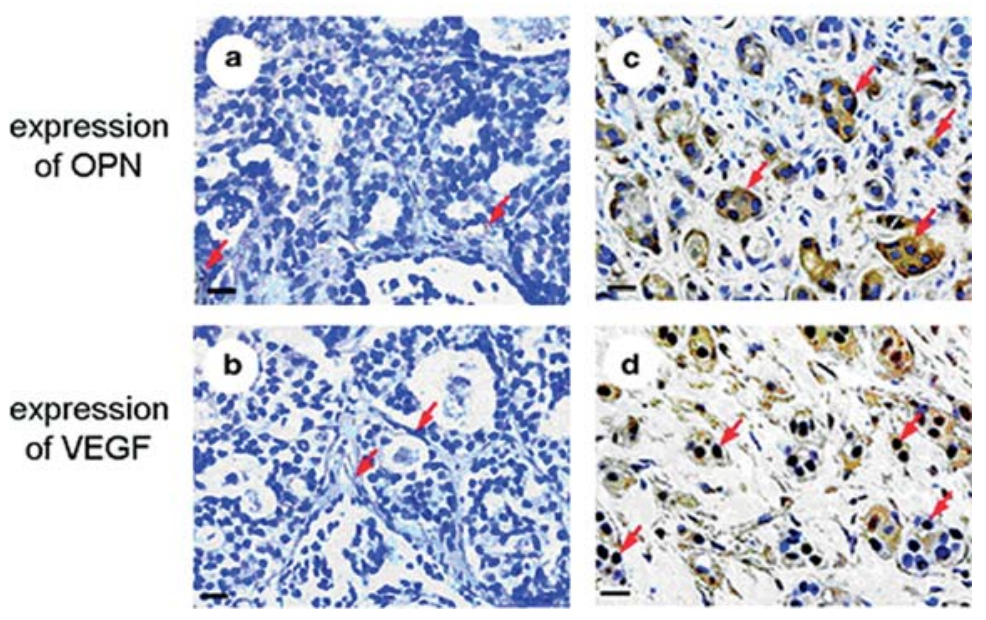

Figure 9. Expression of OPN and VEGF in lung tissue (SGC-OPN- group) or metastasis tumor specimens (SGC7901 group) (red arrows, positive staining; Bar, $20 \mu \mathrm{m})$ 
and NF-кB in SGC7901 cells also need to be explored in future studies. Our study revealed that down-regulation of OPN inhibited tumor growth and metastases of SGC7901 cells in mice, and improved mouse survival rate compared to controls. Induction of VEGF overexpression may be an important mechanism by which OPN is involved in gastric metastases. We hypothesized that overexpression of OPN and VEGF in patients with advanced gastric cancer resulted in tumor angiogenesis. Of note, bevacizumab, a recombinant, humanized monoclonal antibody that targets VEGF, has been safely used as a chemotherapy and is active in the treatment of advanced gastric cancer (19). It is therefore considered that inhibition of both VEGF and OPN may be a more rational strategy to treat gastric cancer.

\section{Acknowledgements}

This study was supported by grants from Shanghai Medical College, Fudan University, China.

\section{References}

1. Kamangar F, Dores GM and Anderson WF: Patterns of cancer incidence, mortality, and prevalence across five continents: defining priorities to reduce cancer disparities in different geographic regions of the world. J Clin Oncol 24: 2137-2150, 2006.

2. Rangaswami H, Bulbule A and Kundu GC: Osteopontin: role in cell signaling and cancer progression. Trends Cell Biol 16 79-87, 2006.

3. Dai N, Bao Q, Lu A and Li J: Protein expression of osteopontin in tumor tissues is an independent prognostic indicator in gastric cancer. Oncology 72: 89-96, 2007.

4. Tang H, Wang J, Bai F, et al: Positive correlation of osteopontin, cyclooxygenase- 2 and vascular endothelial growth factor in gastric cancer. Cancer Invest 26: 60-67, 2008.

5. Wu CY, Wu MS, Chiang EP, et al: Elevated plasma osteopontin associated with gastric cancer development, invasion and survival. Gut 56: 782-789, 2007.
6. Wai PY, Mi Z, Guo H, et al: Osteopontin silencing by small interfering RNA suppresses in vitro and in vivo CT26 murine colon adenocarcinoma metastasis. Carcinogenesis 26: 741-751, 2005.

7. Liu TS, Wang JY and Chen SY: Down-regulation of osteopontin by siRNA on the biological behavior of gastric cancer cell lines. Chin J Digest 27: 736-740, 2007.

8. Gong M, Lu Z, Fang G, Bi J and Xue X: A small interfering RNA targeting osteopontin as gastric cancer therapeutics. Cancer Lett 272: 148-159, 2008.

9. Hirama M, Takahashi F, Takahashi K, et al: Osteopontin overproduced by tumor cells acts as a potent angiogenic factor contributing to tumor growth. Cancer Lett 198: 107-117, 2003.

10. Cui R, Takahashi F, Ohashi R, et al: Abrogation of the interaction between osteopontin and alphavbeta3 integrin reduces tumor growth of human lung cancer cells in mice. Lung Cancer 57: 302-310, 2007.

11. Du XL, Jiang T, Sheng XG, Gao R and Li QS: Inhibition of osteopontin suppresses in vitro and in vivo angiogenesis in endometrial cancer. Gynecol Oncol 115: 371-376, 2009.

12. Chakraborty G, Jain S and Kundu GC: Osteopontin promotes vascular endothelial growth factor-dependent breast tumor growth and angiogenesis via autocrine and paracrine mechanisms. Cancer Res 68: 152-161, 2008.

13. Bergers $G$ and Benjamin LE: Tumorigenesis and the angiogenic switch. Nat Rev Cancer 3: 401-410, 2003.

14. Khodavirdi AC, Song Z, Yang S, et al: Increased expression of osteopontin contributes to the progression of prostate cancer. Cancer Res 66: 883-888, 2006.

15. Rittling SR and Novick KE: Osteopontin expression in mammary gland development and tumorigenesis. Cell Growth Differ 8. 1061-1069, 1997

16. Ye QH, Qin LX, Forgues M, et al: Predicting hepatitis B viruspositive metastatic hepatocellular carcinomas using gene expression profiling and supervised machine learning. Nat Med 9: 416-423, 2003

17. Sun BS, Dong QZ, Ye QH, et al: Lentiviral-mediated miRNA against osteopontin suppresses tumor growth and metastasis of human hepatocellular carcinoma. Hepatology 48: 1834-1842, 2008.

18. Tang H, Wang J, Bai F, et al: Inhibition of osteopontin would suppress angiogenesis in gastric cancer. Biochem Cell Biol 85: 103-110, 2007.

19. Shah MA, Ramanathan RK, Ilson DH, et al: Multicenter phase II study of irinotecan, cisplatin, and bevacizumab in patients with metastatic gastric or gastroesophageal junction adenocarcinoma. J Clin Oncol 24: 5201-5206, 2006. 